\title{
Brodalumab Is Associated with High Rates of Complete Clearance and Quality of Life Improvement: A Subgroup Analysis of Patients with Psoriasis and Concomitant Psoriatic Arthritis
}

\author{
Georgios Kokolakis $^{\mathrm{a}}$ Kasper Vadstrup $^{\mathrm{b}}$ Jes B. Hansen ${ }^{\mathrm{b}}$ \\ Jose Manuel Carrascosac \\ aPsoriasis Research and Treatment Centre, Clinic of Dermatology, Venereology and Allergology, Charité - \\ Universitätsmedizin Berlin, Berlin, Germany; 'bEO Pharma A/S, Ballerup, Denmark; 'Department of Dermatology, \\ Hospital Universitari Germans Trias i Pujol, Universitat Autònoma of Barcelona, IGTP, Badalona, Spain
}

\section{Keywords}

Brodalumab · Ustekinumab · Psoriasis · Psoriatic arthritis ·

QoL

\begin{abstract}
Background: Psoriatic arthritis (PsA) is a chronic inflammatory disease associated with psoriasis that significantly impairs physical function and quality of life (QoL). Prompt therapeutic intervention is crucial for limiting PsA progression and preventing disability. Objectives: The aim of this study was to compare the efficacy of brodalumab versus ustekinumab and the impact on QoL in patients with moderate-tosevere plaque psoriasis, by concomitant PsA status. Methods: This post hoc analysis of pooled data from the phase 3 AMAGINE-2 and -3 trials evaluated complete skin clearance (100\% improvement of Psoriasis Area and Severity Index [PASI 100]), improvement in symptom severity (Psoriasis Symptom Inventory [PSI] response), and QoL (Dermatology Life Quality Index [DLQI] score of 0/1) by concomitant PsA status. A competing risk model assessed cumulative incidence over 52 weeks with outcomes of PASI 100 or inadequate response. Results: This analysis included 929 patients
\end{abstract}

with moderate-to-severe psoriasis. Concomitant PsA was present in 79/339 (23\%) and 110/590 (19\%) patients receiving brodalumab $210 \mathrm{mg}$ and ustekinumab, respectively. At Week 52, odds ratios (ORs) (95\% confidence intervals [Cls]) for complete clearance with brodalumab versus ustekinumab were $3.15(1.52-6.55, p=0.0015)$ in patients with concomitant PsA and $3.05(2.19-4.26, p<0.0001)$ in patients without concomitant PsA. Corresponding Week 52 ORs (95\% Cls) for DLQI 0/1 with brodalumab versus ustekinumab were $2.05(1.07-3.90, p=0.0277)$ and $1.83(1.32-2.53, p=0.0002)$; Week 52 ORs ( $95 \%$ Cls) for PSI $\leq 8$ with brodalumab versus ustekinumab were $3.42(1.43-8.18, p=0.0036)$ and 1.40 $(1.01-1.95, p=0.0434)$. The 52-week cumulative incidence of patients achieving PASI 100 was significantly higher for brodalumab versus ustekinumab in patients with concomitant PsA $(p=0.0001)$ and in those without concomitant PsA ( $p<$ $0.0001)$. Conclusions: Treatment with brodalumab rapidly results in high levels of complete and sustained skin clearance and greater cumulative treatment benefit in patients with moderate-to-severe psoriasis versus ustekinumab, regardless of concomitant PsA status.

(c) 2021 The Author(s).

Published by S. Karger AG, Basel karger@karger.com www.karger.com/drm

Karger $\stackrel{\text { ' }}{5}$

GOPEN ACCESS
(C) 2021 The Author(s)

Published by S. Karger AG, Basel

This is an Open Access article licensed under the Creative Commons Attribution-NonCommercial-4.0 International License (CC BY-NC) (http://www.karger.com/Services/OpenAccessLicense), applicable to the online version of the article only. Usage and distribution for commercial purposes requires written permission.
Correspondence to:

Georgios Kokolakis, georgios.kokolakis@ charite.de 


\section{Introduction}

Psoriatic arthritis (PsA) is a chronic inflammatory musculoskeletal disorder estimated to affect up to $40 \%$ of patients with psoriasis [1]. PsA affects both the joints and the entheses and can lead to progressive joint destruction, disability and significantly impaired quality of life (QoL) $[2,3]$. Like psoriasis, PsA is also associated with an increased risk of comorbidities, such as obesity, insulin resistance, inflammatory bowel disease and cardiovascular disease [4-6].

The early diagnosis and treatment of PsA are crucial to reduce the risk of joint damage, disability and comorbidities [7-9]. Since PsA usually occurs after the development of psoriasis [10], it is important that patients with psoriasis are routinely screened for signs of PsA. The therapeutic goals for patients with PsA are to minimise disease activity, prevent disease progression, alleviate symptoms, improve QoL and restore functional ability $[10,11]$. Treatment decisions are based on disease severity, joint involvement and associated skin symptoms [12].

There have been significant advances in our understanding of the pathogenesis of PsA in recent years, which have led to advances in the treatment of the disease, including the introduction of biologics targeting tumour necrosis factor alpha (TNF- $\alpha$ ), interleukin (IL)-12/23, IL$17 \mathrm{~A}$ and IL-23 [13]. Randomised controlled trials have established the efficacy of the anti-IL-12/23 monoclonal antibody ustekinumab in the treatment of patients with active PsA who had previously received conventional and/or anti-TNF biological agents $[14,15]$. More recently, guselkumab, a monoclonal antibody that selectively inhibits IL-23, was approved for the treatment of patients with active PsA [16-18]. However, a significant proportion of patients with PsA fail to have a sustained response to biologics $[6,19]$, and head-to-head studies of biologics in patients with PsA are needed to better inform treatment choices [3].

Brodalumab is a fully human monoclonal antibody with a unique mechanism of action. It binds with high affinity to the IL-17 receptor subunit A (IL-17 RA), thereby inhibiting downstream signalling of multiple IL-17 family cytokines (IL-17A, IL-17F, IL-17C, IL-17E) involved in the pathogenesis of psoriasis [20], unlike other antiIL-17 biologics, such as secukinumab and ixekizumab, which specifically target IL-17A [21, 22]. Brodalumab is currently approved for the treatment of moderate-to-severe plaque psoriasis $[23,24]$ in the USA, EU, Canada and some Asian countries, and for PsA in Japan [25].
Brodalumab demonstrated high rates of total skin clearance, as shown by $100 \%$ reduction in Psoriasis Area and Severity Index (PASI 100), in two phase 3 trials in patients with moderate-to-severe plaque psoriasis: AMAGINE-2 and AMAGINE-3 [26]. Brodalumab has also demonstrated efficacy relative to placebo in phase 2 and 3 trials in patients with PsA $[6,27]$. The aim of this post hoc analysis was to evaluate the efficacy of brodalumab compared to ustekinumab and the impact on patient QoL through 52 weeks in patients with moderateto-severe plaque psoriasis, with or without concomitant PsA, using pooled data from the phase 3 AMAGINE-2 and AMAGINE-3 trials.

\section{Materials and Methods}

\section{Study Design and Patients}

The AMAGINE-2 (NCT01708603) and AMAGINE-3 (NCT01708629) trial designs have been previously reported in detail [26] and are illustrated in online supplementary Figure S1 (see www.karger.com/doi/10.1159/000520290 for all online suppl. material). Briefly, patients $\geq 18$ years of age with moderate-to-severe plaque psoriasis (defined as a PASI score $\geq 12$, static Physician's Global Assessment [sPGA] score of $\geq 3$ and $\geq 10 \%$ body surface area involvement of $\geq 6$ months' duration) were randomised 2:2:1:1 to receive brodalumab $210 \mathrm{mg}$, brodalumab $140 \mathrm{mg}$, ustekinumab (45 mg for patients with a body weight $\leq 100 \mathrm{~kg}$ and $90 \mathrm{mg}$ for patients $>100 \mathrm{~kg}$ on Day 1 , Week 4 and every 12 weeks [Q12W] thereafter) or placebo on Day 1 and Weeks 1, 2, 4, 6, 8 and 10. At Week 12 , patients who were originally randomised to brodalumab were re-randomised 2:2:2:1 to receive a brodalumab maintenance regimen of $210 \mathrm{mg}$ once every 2 weeks (Q2W) or $140 \mathrm{mg}$ Q2W, every 4 weeks (Q4W) or every 8 weeks (Q8W). Placebo patients received $210 \mathrm{mg}$ of brodalumab Q2W, and ustekinumab patients continued to receive ustekinumab Q12W until Week 52.

Patients who had an inadequate response (defined as sPGA $\geq 3$ or persistent values of 2 over $\mathrm{a} \geq 4$-week period at, or after, Week 16) received rescue treatment with brodalumab $210 \mathrm{mg}$. After Week 16 and through Week 52, patients receiving brodalumab were rescued with brodalumab $210 \mathrm{mg}$ Q2W, while those receiving ustekinumab remained on ustekinumab except at Week 16, when they could receive rescue treatment (210 mg of brodalumab Q2W). Rescue treatment was blinded. Patients who did not respond to rescue treatment for $\geq 12$ weeks had treatment discontinued.

The AMAGINE-2 and AMAGINE- 3 trials were conducted in accordance with the International Conference on Harmonisation for Good Clinical Practice, the Declaration of Helsinki, and the Health Insurance Portability and Accountability Act of 1996 or relevant regional regulations. The institutional review boards at each participating centre approved the study protocols.

\section{Assessments}

Concomitant PsA Status

Concomitant PsA status was defined by medical history and categorised in yes/no options. 
Table 1. Baseline demographic and disease characteristics (pooled data by treatment arm and concomitant PsA status from the AMAGINE-2 and -3 trials)

\begin{tabular}{|c|c|c|c|c|}
\hline & \multicolumn{2}{|c|}{ Brodalumab $(n=339)$} & \multicolumn{2}{|c|}{ Ustekinumab $(n=590)$} \\
\hline Female, $n(\%)$ & $32.0(40.5)$ & $77.0(29.6)$ & $42.0(38.2)$ & $144.0(30.0)$ \\
\hline Age, mean (SD), years & $49.8(13.5)$ & $43.0(13.0)$ & $48.3(11.7)$ & $44.4(13.3)$ \\
\hline Weight, mean (SD), kg & $92.8(26.3)$ & $89.6(23.5)$ & $91.2(24.4)$ & $91.0(22.5)$ \\
\hline Prior biologic failure, $n(\%)$ & $18.0(40.0)$ & $28.0(54.9)$ & $19.0(38.8)$ & $41.0(38.7)$ \\
\hline Prior systemic use, $n(\%)$ & $61.0(77.2)$ & $133.0(51.2)$ & $78.0(70.9)$ & $262.0(54.6)$ \\
\hline $\mathrm{CRP}$, mean (SD), mg/L & $11.9(17.2)$ & $5.3(9.9)$ & $9.7(20.1)$ & $4.5(5.5)$ \\
\hline PASI, mean (SD) & $21.3(8.0)$ & $20.2(7.8)$ & $21.4(9.0)$ & $19.7(8.2)$ \\
\hline DLQI, mean (SD) & $15.9(7.3)$ & $14.4(7.2)$ & $14.6(7.2)$ & $14.9(7.3)$ \\
\hline
\end{tabular}

CRP, C-reactive protein; DLQI, Dermatology Life Quality Index; PASI, Psoriasis Area and Severity Index; PsA, psoriatic arthritis; SD, standard deviation.

Disease Severity, Response and QoL

Disease severity was evaluated using the PASI and the Psoriasis Symptom Inventory (PSI). The PASI is the most widely used outcome measure for assessing disease severity in clinical trials of biologics to treat psoriasis [28]. The PSI is an 8-point, patient-reported outcome measure, which was developed by Amgen, for assessing the severity of psoriasis symptoms (i.e., itch, redness, scaling, burning, stinging, cracking, flaking and pain), with each symptom rated as either 0 (not at all severe), 1 (mild), 2 (moderate), 3 (severe) or 4 (very severe) [29]. PSI was assessed daily from screening through Week 24 and Weeks 48-52 and aggregated to a weekly score for patients with at least 4 values within that week. A PSI responder was defined as having a total score $\leq 8$ and no item $>1$ throughout the week using 24-hour recall.

QoL was evaluated using the Dermatology Life Quality Index (DLQI). The DLQI consists of 10 questions covering 6 domains: symptoms and feelings, daily activities, leisure, work and school, personal relationships and bother with treatment. A score of 0 or 1 indicates no effect at all on a patient's life [30]. DLQI was assessed at Weeks 0, 2, 4 and every fourth week thereafter.

Responder Analyses for Clearance (PASI 100), PSI Responder Status and DLQI 0/1 at a Given Time Point (Weeks 0-52) by Concomitant PsA Status

Data from patients randomised to receive constant dosing of either the approved dose of brodalumab (210 mg Q2W) or ustekinumab for the entire 52-week treatment period, subdivided according to concomitant PsA status at baseline, were included in this analysis. The proportions of patients achieving PASI 100, PSI responder status and DLQI 0/1 response were compared according to concomitant PsA status and visit. Comparisons between treatment groups were reported as odds ratios (ORs) and 95\% confidence intervals (CIs) calculated using the Cochran-MantelHaenszel method and adjusted for study, baseline total body weight group ( $\leq 100 \mathrm{~kg},>100 \mathrm{~kg}$ ), geographical region, and within study and subgroup baseline score ( $\leq$ median, $>$ median).
Cumulative Benefit through 52 Weeks by Concomitant PsA Status

Cumulative benefit for each endpoint was determined as the area under the curve (AUC) over 52 weeks for PASI and change in DLQI and 24 weeks for PSI responder status by concomitant PsA status. The AUC was calculated using the trapezoidal rule:

$$
\text { Total AUC }=\sum_{\mathrm{i}}^{N}={ }_{0} \frac{1}{2}\left(\mathrm{P}_{\mathrm{i}}+\mathrm{P}_{\mathrm{i}+1}\right)\left(\mathrm{T}_{\mathrm{i}+1}+\mathrm{T}_{\mathrm{i}}\right),
$$

where $N=$ number of assessment time points, $0=$ baseline, $\mathrm{P}_{\mathrm{i}}=$ percentage of responders, $\mathrm{T}_{\mathrm{i}}=$ time point.

Cumulative benefit was normalised to a percentage of the maximum possible nominal AUC of 5,200 for PASI 100 and DLQI 0/1 (based on a 100\% response rate over 52 weeks) and 2,400 for PSI responder status (based on a 100\% response rate over the first 24 weeks). A $t$ test was used to test $p$ values for treatment differences of brodalumab versus ustekinumab. AUC ratios (brodalumab total AUC value divided by ustekinumab total AUC value) were calculated, with values $>1.0$ indicating a greater clinical benefit for brodalumab versus ustekinumab, and 95\% CIs were calculated via bootstrapping.

Cumulative Incidence of Complete Clearance over 52 Weeks by Concomitant PsA Status

Cumulative incidence of complete clearance over 52 weeks was analysed by concomitant PsA status using a Competing Risk model [31] with the outcome of (a) achieving PASI 100 or (b) inadequate response (defined as $s P G A \geq 3$ or $s P G A \geq 2$ for more than 4 weeks) at, or after, Week 16. Comparisons between treatment arms were performed using subdistribution hazard ratios (HRs) and associated $\chi^{2}$ tests $[32,33]$ and adjusted for baseline characteristics, as detailed for the responder analyses. Non-responder imputation was used to handle missing data. 


\section{Results}

\section{Baseline Demographic and Disease Characteristics}

A total of 929 patients (brodalumab $210 \mathrm{mg}, n=339$; ustekinumab, $n=590$ ) were included in this analysis. Concomitant PsA was present in $23 \%$ and $19 \%$ of patients receiving brodalumab and ustekinumab, respectively. Baseline demographic and disease characteristics were generally balanced across treatments (Table 1). For both the brodalumab and ustekinumab treatment groups, patients with concomitant PsA had longer disease duration, higher levels of C-reactive protein, and were more likely to have prior biologic experience compared with patients without PsA (Table 1).

\section{Efficacy}

Responder Analysis for Complete Clearance (PASI 100), PSI and DLQI 0/ 1 by Concomitant PsA Status

Treatment with brodalumab resulted in significantly more patients achieving complete clearance (PASI 100) compared with ustekinumab, from Week 4 through Week 52 for patients without PsA $(p<0.001)$ and from Week 6 through Week 52 for patients with PsA $(p<0.05)$ (Fig. 1a). ORs (95\% CIs) for complete clearance with brodalumab versus ustekinumab at Week 52 were: with concomitant PsA, OR $3.15(1.52-6.55, p=0.0015)$; without concomitant PsA, OR $3.05(2.19-4.26, p<0.0001)$.

Treatment with brodalumab also resulted in significantly more patients achieving DLQI 0/1 compared with ustekinumab, from Week 2 through Week 52 for patients without PsA $(p<0.05)$. In patients with PsA, more patients achieved DLQI 0/1 compared with ustekinumab from Week 4 through Week 52, although significance was not reached at Weeks 16, 20, 24 and 32 due to lower numbers of patients in the compared brodalumab and ustekinumab groups (Fig. 1b). ORs (95\% CIs) for DLQI 0/1 with brodalumab versus ustekinumab at Week 52 were: with concomitant PsA, OR $2.05(1.07-3.90, p=0.0277)$; without concomitant PsA, OR $1.83(1.32-2.53, p=0.0002)$.

Treatment with brodalumab also resulted in more patients achieving PSI responder status compared with ustekinumab from Week 2 through Week 52 in both groups, although significance was not reached at Weeks 14, 17, 19, 21 and 49 in the group with PsA and at Week 49 in the group without PsA due to lower numbers of patients in the compared brodalumab and ustekinumab groups (Fig. 1c). ORs (95\% CIs) for PSI responder status with brodalumab versus ustekinumab at Week 52 were: with concomitant PsA, OR $3.42(1.43-8.18, p=0.0036)$; without concomitant PsA, OR $1.40(1.01-1.95, p=0.0434)$.
Cumulative Benefit through 52 Weeks by

Concomitant PsA Status

Brodalumab treatment resulted in significantly greater cumulative benefit (PASI 100) compared with ustekinum$\mathrm{ab}$ in the subgroups with and without concomitant PsA (Fig. 2a). AUC ratios were $1.84(p=0.0001)$ and $1.68(p<$ $0.0001)$, respectively, in the subgroups with and without concomitant PsA.

Significant differences in cumulative treatment benefit favouring brodalumab versus ustekinumab were also observed for DLQI 0/1 (Fig. 2b). AUC ratios were 1.40 ( $p=$ $0.0016)$ and $1.33(p<0.0001)$, respectively, in the subgroups with and without concomitant PsA.

Similar findings were observed with respect to achievement of PSI responder status up to Week 24 (Fig. 2c). AUC ratios were $1.49(p=0.0005)$ and $1.34(p<0.0001)$, respectively, in the subgroups with and without concomitant PsA.

Competing Risk Model for Achieving Skin Clearance

The competing risk analysis of either achieving PASI 100 or having an inadequate response over 52 weeks showed that brodalumab patients with concomitant PsA were significantly more likely to achieve PASI 100 at least once over 52 weeks than ustekinumab patients, with $70.1 \%$ (95\% CI, 60.4-81.5\%) of responders on brodalumab versus $43.9 \%$ (95\% CI, 35.4-54.3\%) of responders on ustekinumab (HR 2.34; 95\% CI, 1.61-3.38; $p<0.0001$ ) (Fig. 3b). In patients without concomitant PsA, 76.1\% (95\% CI, 71.0-81.5\%) of brodalumab patients achieved PASI 100 versus $53.2 \%$ (95\% CI, 48.9-57.9\%) of ustekinumab patients (HR 2.07; 95\% CI, 1.71-2.50; $p<$ 0.0001) (Fig. 3a). Furthermore, there were fewer inadequate responders to brodalumab compared with ustekinumab (Fig. 3a,b).

The median time to complete clearance was 13 weeks for brodalumab versus 36 weeks for ustekinumab in patients without PsA (Fig. 3a). In patients with concomitant PsA, the median time to complete clearance was 15 weeks for brodalumab but was indeterminable for ustekinumab, as less than $50 \%$ of patients achieved complete clearance by Week 52 (Fig. 3b).

\section{Discussion/Conclusion}

Previous studies have demonstrated the efficacy of brodalumab in patients with PsA $[6,27]$. Brodalumab (140 mg or $280 \mathrm{mg}$ Q2W) significantly improved clinical response rates versus placebo in patients with PsA in a 


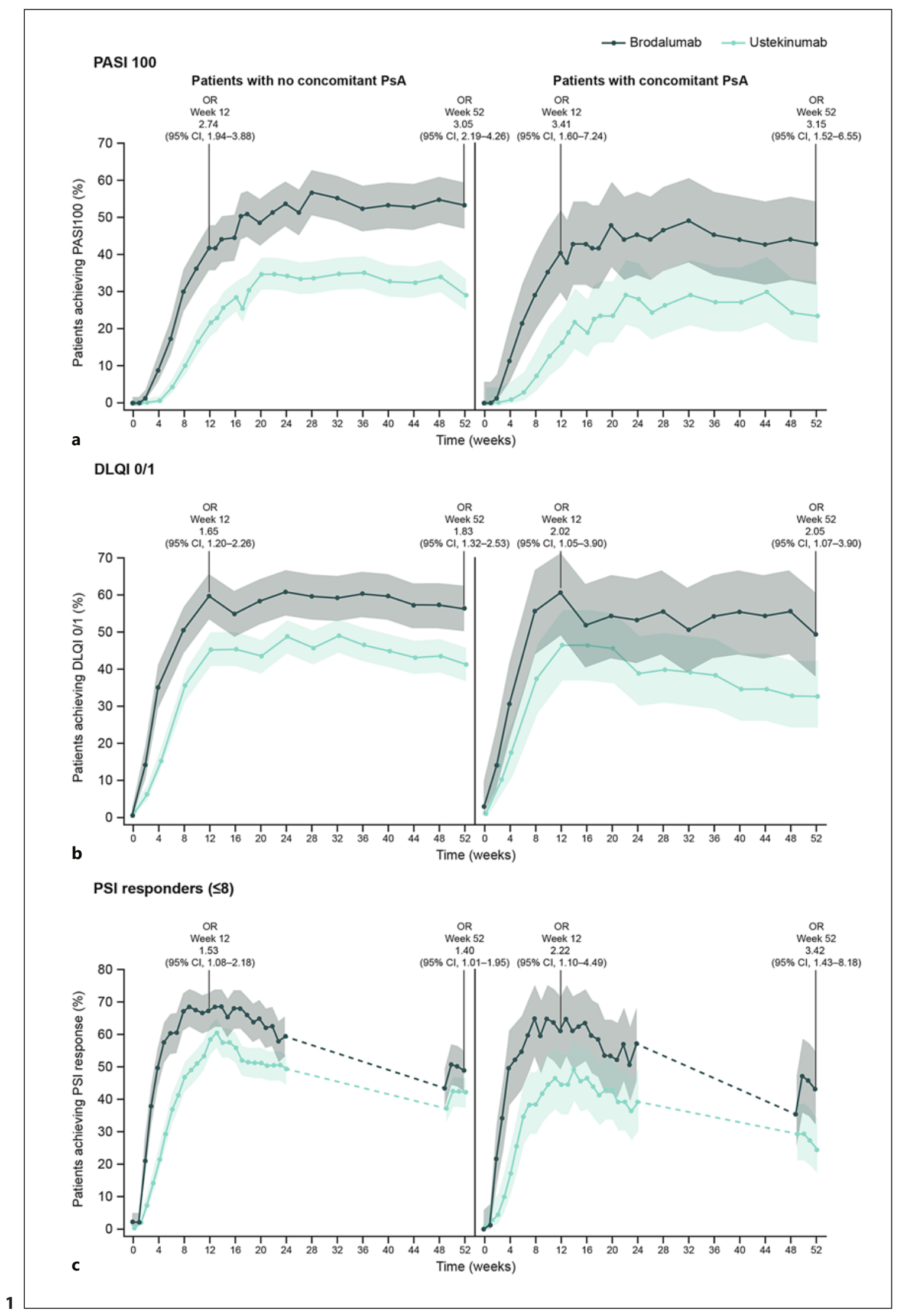

(For legend see next page.) 


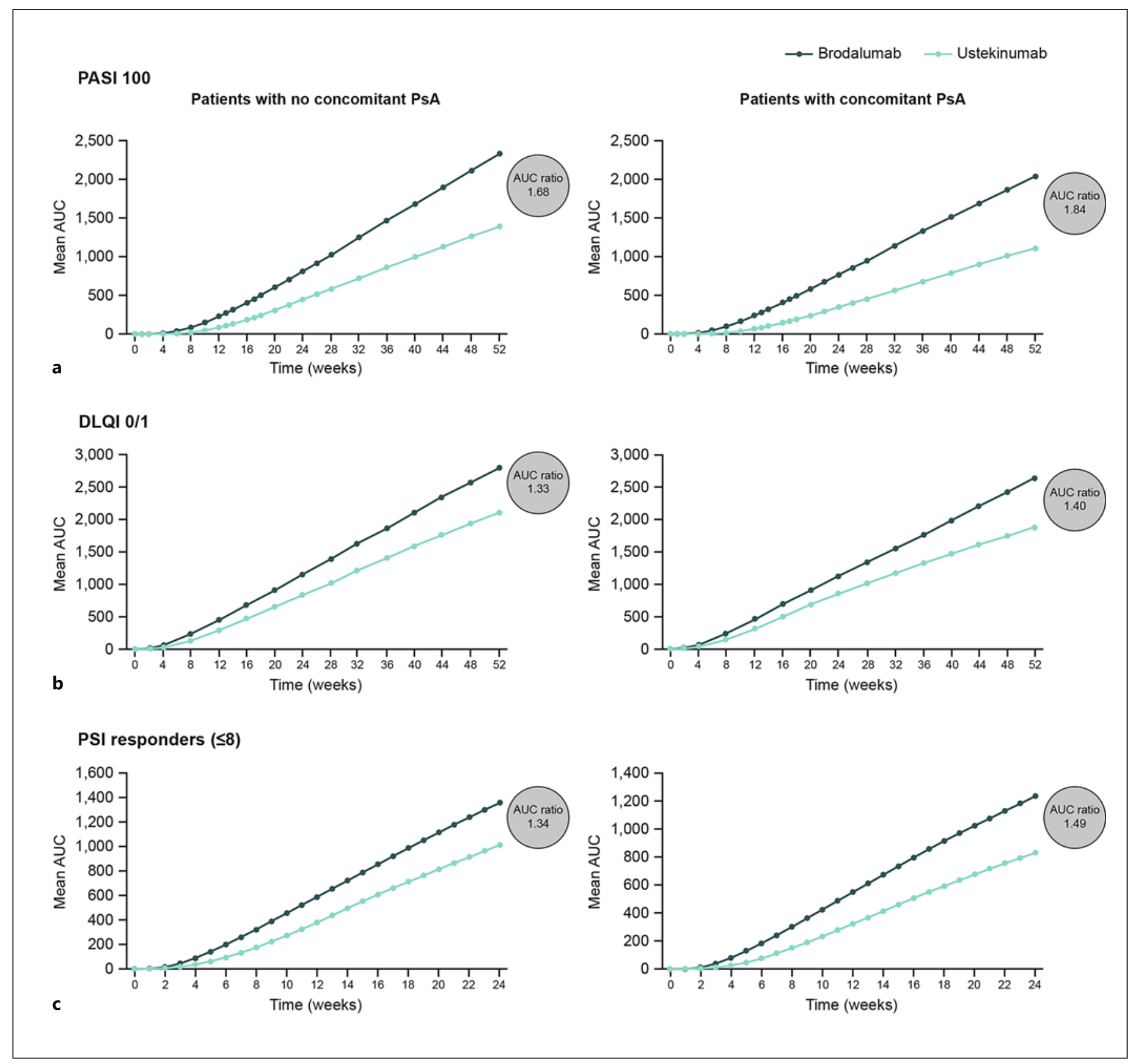

Fig. 2. Cumulative benefit through 52 weeks with respect to PASI 100 (a), DLQI 0/1 (b) and PSI responder $(\leq 8)$ status (c), by visit, treatment and concomitant PsA status (NRI). AUC, area under the curve; DLQI, Dermatology Life Quality Index; NRI, non-responder imputation; PASI, Psoriasis Area and Severity Index; PsA, psoriatic arthritis; PSI, Psoriasis Symptom Inventory.

Fig. 1. Percentage of patients achieving PASI 100 (a), DLQI $0 / 1(\mathbf{b})$ and PSI responder $(\leq 8)$ status (c), by visit, treatment and concomitant PsA status (NRI). ${ }^{a}$ Shading indicates 95\% CI. PSI data were not collected between

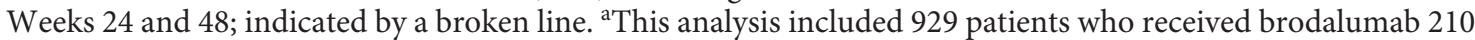
$\mathrm{mg}(n=339)$ or ustekinumab $(n=590)$. Of the patients who received brodalumab, 79 had concomitant PsA and 260 had no concomitant PsA; of those who received ustekinumab, 110 had concomitant PsA and 480 had no concomitant PsA. CI, confidence interval; DLQI, Dermatology Life Quality Index; NRI, non-responder imputation; OR, odds ratio; PASI, Psoriasis Area and Severity Index; PsA, psoriatic arthritis; PSI, Psoriasis Symptom Inventory.

Complete Skin Clearance with Brodalumab in Psoriasis and PsA
Dermatology 2022;238:620-629 DOI: $10.1159 / 000520290$ 
Patients with no concomitant PsA

Brodalumab

Ustekinumab

$\mathrm{HR}$

$(95 \% \mathrm{Cl})$
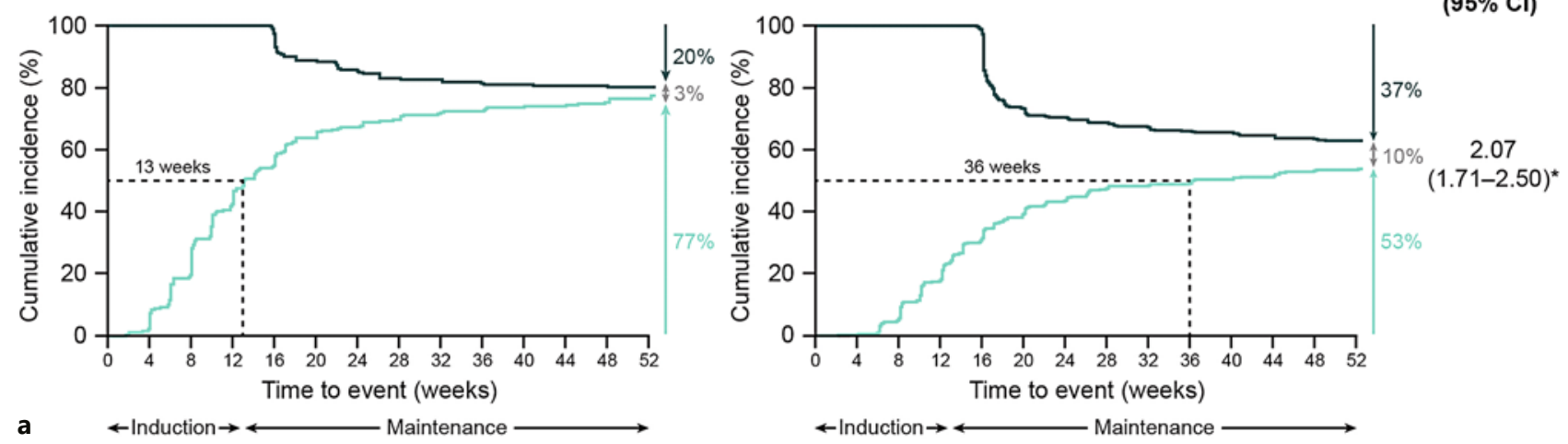

a

$\leftarrow$ Induction $\rightarrow \longleftarrow$ Maintenance

$\leftarrow$ Induction $\rightarrow \longleftarrow$ Maintenance

Patients with concomitant PsA

Brodalumab

Ustekinumab

HR
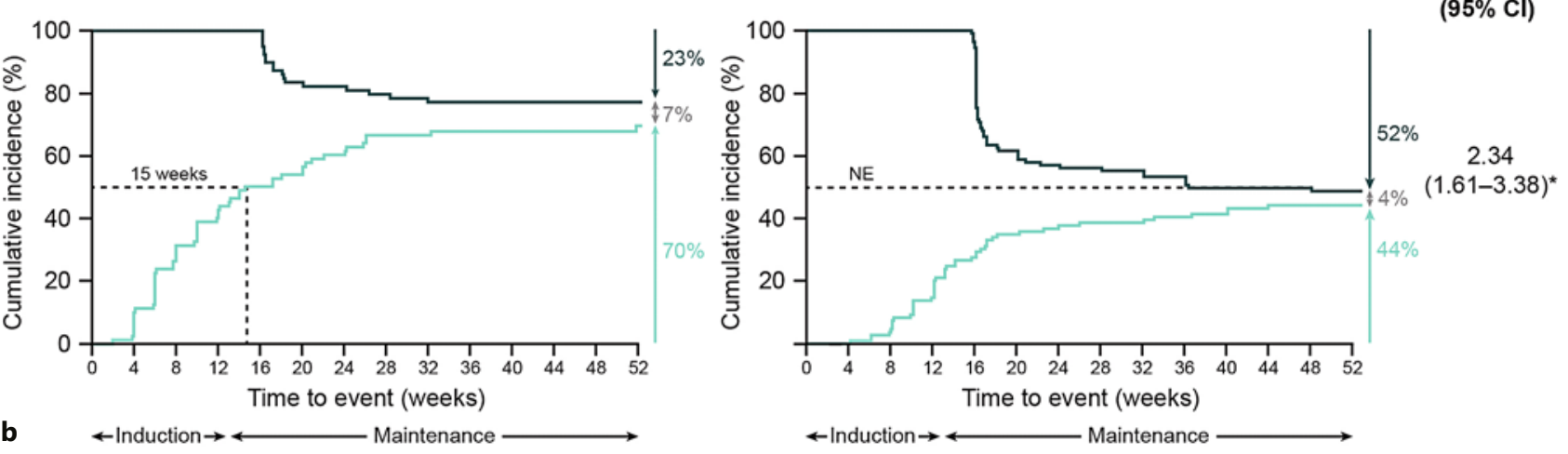

b

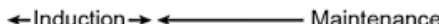

- Complete clearance - Inadequate response ${ }^{a} \leftrightarrow$ Never achieved complete clearance $^{a}$

.... Time to achievement of complete clearance by $50 \%$ of patients

Fig. 3. Cumulative incidence of patients achieving PASI 100, by visit, treatment and concomitant PsA status (competing risk analysis) (NRI). ${ }^{*} p<0.0001$. ${ }^{a}$ Defined as static Physician's Global Assessment $\geq 3$ or persistent values of 2 over at least a 4 -week period or after Week 16. CI, confidence interval; HR, hazard ratio; NE, not estimable; NRI, non-responder imputation; PsA, psoriatic arthritis.

phase 2, randomised, double-blind, placebo-controlled trial [6]. The efficacy and safety of brodalumab in adults with active PsA and inadequate response, or intolerance, to conventional treatment were further evaluated in the phase 3 AMVISION-1 and AMVISION-2 trials. Brodalumab (140 mg or $210 \mathrm{mg}$ once Q2W) was associated with rapid and significant improvements in signs and symptoms of PsA versus placebo [27].
In addition to musculoskeletal symptoms, cutaneous manifestations and severity of psoriasis are also important considerations when making treatment decisions for patients with PsA $[12,34]$. In this pooled analysis of data from the phase 3 AMAGINE-2 and AMAGINE-3 trials, we assessed the reduction in disease severity and impact on patient QoL through 52 weeks, in patients with moderate-to-severe psoriasis, by presence or not of concomitant PsA. We found that brodalumab (210 mg once Q2W) 
rapidly achieved higher levels of complete skin clearance (PASI 100), was significantly better at improving QoL and reducing patient-reported symptoms of psoriasis, and resulted in a greater cumulative treatment benefit through 52 weeks in patients with moderate-to-severe psoriasis than ustekinumab, regardless of concomitant PsA status.

In an earlier analysis of data from AMAGINE-2 and AMAGINE-3, brodalumab showed significantly higher PASI 75, 90 and 100 response rates versus ustekinumab [26]. In our analysis, a significantly higher proportion of brodalumab-treated than ustekinumab-treated patients achieved rapid and consistent complete skin clearance over the 52-week treatment period, with significant differences observed from Week 4 onwards for patients without PsA and from Week 6 onwards for patients with concomitant PsA. In addition to comparing the response to brodalumab versus ustekinumab at specific time points, we examined the cumulative treatment benefit and cumulative incidence of response to each drug over 52 weeks of treatment. Cumulative measures of drug efficacy - which account for speed, durability and magnitude of treatment responses - have been suggested to be particularly relevant for psoriasis and other chronic diseases that have continuous impact on patients [35]. These analyses demonstrated that brodalumab was associated with a greater cumulative benefit and a higher likelihood of achieving PASI 100 at least once over 52 weeks compared to ustekinumab, and that the differences were more prominent among patients with concomitant PsA than those without. Indeed, while $50 \%$ of brodalumab-treated patients with PsA had achieved PASI 100 at least once by week 15, there was no time through Week 52 at which $50 \%$ of ustekinumab-treated patients with PsA achieved PASI 100.

Achievement of PASI 100 was associated with improvement in QoL, as measured by DLQI 0/1, in patients with or without concomitant PsA. These findings reflect those of Warren et al. [36] who recently reported that improvement in QoL, as measured by DLQI 0/1, was associated with achievement of PASI 100 in patients receiving brodalumab. Furthermore, the improvement in QoL observed in our study was associated with a rapid reduction in the severity of psoriasis symptoms, as measured by PSI response. The likelihood of achieving PSI responder status favoured brodalumab in patients with or without concomitant PsA, although ORs were noticeably higher in the group with PsA, at both Weeks 12 and 52 .

These data add to the growing body of evidence from clinical-trial and real-world settings suggesting that com- plete skin clearance is associated with substantially reduced physical burden of psoriasis, increased treatment satisfaction and improved QoL [37-42] and that rapid resolution of psoriasis symptoms correlates with improvements in QoL $[43,44]$.

Patients with PsA are considered high-need because of progressive joint symptoms/pain and disability [45]. Early diagnosis of PsA enables prompt treatment initiation, resulting in improved clinical outcomes and reduced disease severity and joint damage $[45,46]$. Delays in diagnosis increase the risk of joint damage and poor long-term physical function [47]. As patients with psoriasis and without current PsA might go on to develop PsA [48] and because brodalumab is effective at treating psoriasis in patients with or without symptoms of PsA, early treatment of psoriasis with brodalumab might potentially prevent the evolution of PsA in asymptomatic patients.

This study had some limitations. Firstly, analyses were of pooled data from clinical trials not designed and statistically powered to assess these specific endpoints. Secondly, the data were from a clinical trial population with strict inclusion and exclusion criteria and may therefore not be representative of real-world patient populations. Additionally, concomitant PsA was defined by medical history, and details related to PsA clinical characteristics and treatment were not collected. Notably, it is likely that only patients with well-controlled PsA entered the AMAGINE-2 and AMAGINE-3 studies, resulting in a limited number of patients with psoriasis with concomitant PsA being included. Finally, analyses were restricted to patients in constant treatment arms, further reducing the number of patients included.

In conclusion, the results from this analysis of pooled data from the phase 3 AMAGINE-2 and AMAGINE-3 trials demonstrate that treatment with brodalumab rapidly results in high levels of complete and sustained skin clearance and greater cumulative treatment benefit in patients with moderate-to-severe psoriasis than treatment with ustekinumab, regardless of concomitant PsA status. Thus, brodalumab may offer an additional treatment strategy for patients with psoriasis with concomitant PsA.

\section{Key Message}

Brodalumab achieved PASI 100 and improved QoL in patients with psoriasis with and without concomitant PsA. 


\section{Acknowledgement}

Medical writing support was provided by Susan Dyas, MSc, of Adelphi Communications Limited, Macclesfield, UK, and Clair Geary, PhD, of Alphabet Health, New York, NY, funded by LEO Pharma A/S, in accordance with Good Publication Practice (GPP3) guidelines.

\section{Statement of Ethics}

The AMAGINE-2 and AMAGINE-3 trials were conducted in accordance with the International Conference on Harmonisation for Good Clinical Practice, the Declaration of Helsinki, and the Health Insurance Portability and Accountability Act of 1996 or relevant regional regulations. The institutional review boards at each participating centre approved the study protocols. All patients provided written informed consent before the initiation of study procedures.

\section{Conflict of Interest Statement}

G.K. has received honoraria for participation in advisory boards, in clinical trials and/or as speaker from AbbVie Deutschland $\mathrm{GmbH} \& \mathrm{Co}$. KG, Abbott GmbH, Actelion Pharmaceuticals Ltd., AMGEN GmbH, Basilea Pharmaceutica Ltd., Bayer AG, Biogen IDEC GmbH, Celgene GmbH, Hexal-Sandoz GmbH, JanssenCilag GmbH, LEO Pharma GmbH, Lilly Deutschland $\mathrm{GmbH}$,
MSD Sharp \& Dohme GmbH, Novartis Pharma GmbH, Parexel International $\mathrm{GmbH}$, Pfizer Deutschland $\mathrm{GmbH}$ and UCB Pharma GmbH. J.B.H. and K.V. are employees of LEO Pharma. J.M.C. has received honoraria for participation in advisory boards, in clinical trials and/or as speaker from AbbVie, AMGEN, Biogen, Celgene, Janssen-Cilag, LEO Pharma, Lilly, Novartis Pharma $\mathrm{GmbH}$, Pfizer, UCB, Sandoz, Mylan and Almirall.

\section{Funding Sources}

The brodalumab clinical study programme was sponsored by Amgen/AstraZeneca; this analysis was performed by LEO Pharma A/S.

\section{Author Contributions}

All named authors meet the International Committee of Medical Journal Editors (ICMJE) criteria for authorship for this article, take responsibility for the integrity of the work as a whole, and have given their approval for this version to be published.

\section{Data Availability Statement}

The datasets generated during and/or analysed during the current study are available from the corresponding author on reasonable request for non-commercial purposes.

\section{References}

1 Gladman DD, Antoni C, Mease P, Clegg DO, Nash P. Psoriatic arthritis: epidemiology, clinical features, course, and outcome. Ann Rheum Dis. 2005;64(Suppl 2):ii14-7.

2 Griffiths CEM, Jo SJ, Naldi L, Romiti R, Guevara-Sangines $\mathrm{E}$, Howe $\mathrm{T}$, et al. A multidimensional assessment of the burden of psoriasis: results from a multinational dermatologist and patient survey. Br J Dermatol. 2018; 179(1):173-81.

3 Mahmood F, Coates LC, Helliwell PS. Current concepts and unmet needs in psoriatic arthritis. Clin Rheumatol. 2018;37(2):297305.

4 Ferguson LD, Siebert S, McInnes IB, Sattar N. Cardiometabolic comorbidities in RA and PsA: lessons learned and future directions. Nat Rev Rheumatol. 2019;15(8):461-74.

5 Gudu T, Gossec L. Quality of life in psoriatic arthritis. Expert Rev Clin Immunol. 2018; 14(5):405-17.

6 Mease PJ, Genovese MC, Greenwald MW, Ritchlin CT, Beaulieu AD, Deodhar A, et al. Brodalumab, an anti-IL17RA monoclonal antibody, in psoriatic arthritis. N Engl J Med. 2014;370(24):2295-306.
7 McArdle A, Pennington S, FitzGerald O. Clinical features of psoriatic arthritis: a comprehensive review of unmet clinical needs. Clinic Rev Allerg Immunol. 2018;55(3):27194.

8 Mease PJ, Gladman DD, Papp KA, Khraishi MM, Thaçi D, Behrens F, et al. Prevalence of rheumatologist-diagnosed psoriatic arthritis in patients with psoriasis in European/North American dermatology clinics. J Am Acad Dermatol. 2013;69(5):729-35.

9 Schmidt A, Glimm AM, Haugen IK, Hoff P, Schmittat G, Burmester GR, et al. Detection of subclinical skin manifestation in patients with psoriasis and psoriatic arthritis by fluorescence optical imaging. Arthritis Res Ther. 2020;22(1):192.

10 Ocampo DV, Gladman D. Psoriatic arthritis. F1000Res. 2019;8:F1000 Faculty Rev1665.

11 Costa L, Perricone C, Chimenti MS, Del Puente A, Caso P, Peluso R, et al. Switching between biological treatments in psoriatic arthritis: a review of the evidence. Drugs R D. 2017;17(4):509-22.

12 Kavanaugh A, Coates LC, van der Windt DA, Corp N, Soriano ER. GRAPPA treatment recommendations: updates and methods. J Rheumatol Suppl. 2020;96:41-5.
13 Ghoreschi K, Balato A, Enerbäck C, Sabat R Therapeutics targeting the IL-23 and IL-17 pathwayin psoriasis. Lancet. 2021;397(10275): 754-766.

14 McInnes IB, Kavanaugh A, Gottlieb AB, Puig L, Rahman P, Ritchlin C, et al. Efficacy and safety of ustekinumab in patients with active psoriatic arthritis: 1 year results of the phase 3 , multicentre, double-blind, placebo-controlled PSUMMIT 1 trial. Lancet. 2013; 382(9894):780-9.

15 Ritchlin C, Rahman P, Kavanaugh A, McInnes IB, Puig L, Li S, et al. Efficacy and safety of the anti-IL-12/23 p40 monoclonal antibody, ustekinumab, in patients with active psoriatic arthritis despite conventional nonbiological and biological anti-tumour necrosis factor therapy: 6-month and 1-year results of the phase 3 , multicentre, double-blind, placebo-controlled, randomised PSUMMIT 2 trial. Ann Rheum Dis. 2014;73(6):990-9.

16 Deodhar A, Helliwell PS, Boehncke WH, Kollmeier AP, Hsia EC, Subramanian RA, et al. Guselkumab in patients with active psoriatic arthritis who were biologic-naive or had previously received TNF $\alpha$ inhibitor treatment (DISCOVER-1): a double-blind, randomised, placebo-controlled phase 3 trial. Lancet. 2020; 395(10230):1115-25. 
17 TREMFYA (guselkumab) approved by U.S. Food and Drug Administration as the first selective interlukin (IL)-23 inhibitor for active psoriatic arthritis [press release, Internet]. [cited Jul 14, 2020]. Available from: https:// www.jnj.com/tremfya-guselkumab-approved-by-u-s-food-and-drug-administration-as-the-first-selective-interleukin-il23-inhibitor-for-active-psoriatic-arthritis.

18 Mease PJ, Rahman P, Gottlieb AB, Kollmeier AP, Hsia EC, Xu XL, et al. Guselkumab in biologic-naive patients with active psoriatic arthritis (DISCOVER-2): a double-blind, randomised, placebo-controlled phase 3 trial. Lancet. 2020;395(10230):1126-36.

19 Megna M, Balato A, Napolitano M, Gallo L, Caso F, Costa L, et al. Psoriatic disease treatment nowadays: unmet needs among the "jungle of biologic drugs and small molecules". Clin Rheumatol. 2018;37(7):1739-41.

20 Russell CB, Rand H, Bigler J, Kerkof K, Timour M, Bautista E, et al. Gene expression profiles normalized in psoriatic skin by treatment with brodalumab, a human anti-IL-17 receptor monoclonal antibody. J Immunol. 2014;192(8):3828-36.

21 Frieder J, Kivelevitch D, Menter A. Secukinumab: a review of the anti-IL-17A biologic for the treatment of psoriasis. Ther Adv Chronic Dis. 2018;9(1):5-21.

22 Syed YY. Ixekizumab: A review in moderate to severe plaque psoriasis. Am J Clin Dermatol. 2017;18(1):147-58.

23 European Medicines Agency. Summary of product characteristics: Kyntheum $210 \mathrm{mg}$ solution for injection in pre-filled syringe [Internet]. 2017. Available from: https://www. ema.europa.eu/en/documents/product-information/kyntheum-epar-product-information_en.pdf.

24 Valeant Pharmaceuticals Inc. Prescribing information: SILIQTM (brodalumab) injection for subcutaneous use [Internet]. 2017. Available from: https://www.accessdata.fda.gov/ drugsatfda_docs/label/2017/761032lbl.pdf.

25 LUMICEF $^{\oplus}$ approved in Japan [press release, Internet]. [Jul 4, 2016]. Available from: https: //www.kyowakirin.com/media_center/ news_releases/2016/e20160704_01.html.

26 Lebwohl M, Strober B, Menter A, Gordon K, Weglowska J, Puig L, et al. Phase 3 studies comparing brodalumab with ustekinumab in psoriasis. N Engl J Med. 2015;373(14):131828.

27 Mease PJ, Helliwell PS, Hjuler KF, Raymond $\mathrm{K}, \mathrm{McInnes} \mathrm{I.} \mathrm{Brodalumab} \mathrm{in} \mathrm{psoriatic} \mathrm{arthri-}$ tis: results from the randomised phase III AMVISION-1 and AMVISION-2 trials. Ann Rheum Dis. 2021;80(2):185-93.

28 Fredriksson T, Pettersson U. Severe psoriasis - oral therapy with a new retinoid. Dermatologica. 1978;157(4):238-44.

29 Bushnell DM, Martin ML, McCarrier K, Gordon K, Chiou CF, Huang X, et al. Validation of the Psoriasis Symptom Inventory (PSI), a patient-reported outcome measure to assess psoriasis symptom severity. J Dermatolog Treat. 2013;24(5):356-60.

30 Finlay AY, Khan GK. Dermatology Life Quality Index (DLQI) - a simple practical measure for routine clinical use. Clin Exp Dermatol. 1994;19(3):210-6.

31 Aalen OO, Johansen S. An empirical transition matrix for non-homogeneous Markov chains based on censored observations. Scan J Stat. 1978;5:141-50.

32 Fine JP, Gray RJ. A proportional hazards model for the subdistribution of a competing risk. J Am Stat Assoc. 1999;94(446):496-509.

33 Gray RJ. A class of K-sample tests for comparing the cumulative incidence of a competing risk. Ann Statist. 1988;16:1141-54.

34 Coates LC, Kavanaugh A, Mease PJ, Soriano ER, Laura Acosta-Felquer M, Armstrong AW, et al. Group for Research and Assessment of Psoriasis and Psoriatic Arthritis 2015 treatment recommendations for psoriatic arthritis. Arthritis Rheumatol. 2016;68(5): 1060-71.

35 Armstrong AW, Feldman SR, Korman NJ, Meng X, Guana A, Nyirady J, et al. Assessing the overall benefit of a medication: cumulative benefit of secukinumab over time in patients with moderate-to-severe plaque psoriasis. J Dermatolog Treat. 2017;28(3):200-5.

36 Warren RB, Hansen JB, Reich K, Paul C, Puig $\mathrm{L}$. Complete clearance and psoriasis area and severity index response for brodalumab and ustekinumab in AMAGINE-2 and -3. J Eur Acad Dermatol Venereol. 2021;35(2):450-7.

37 Feldman SR, Bushnell DM, Klekotka PA, Scanlon M, Martin ML, Wade SW, et al. Differences in psoriasis signs and symptom severity between patients with clear and almost clear skin in clinical practice. J Dermatolog Treat. 2016;27(3):224-7.

38 Girolomoni G, Griffiths CE, Krueger J, Nestle FO, Nicolas JF, Prinz JC, et al. Early intervention in psoriasis and immune-mediated inflammatory diseases: A hypothesis paper. J Dermatolog Treat. 2015;26(2):10312.
39 Lacour JP, Bewley A, Hammond E, Hansen JB, Horne L, Paul C, et al. Association between patient- and physician-reported outcomes in patients with moderate-to-severe plaque Psoriasis treated with Biologics in Real Life (PSO-BIO-REAL). Dermatol Ther (Heidelb). 2020;10(5):1099-109.

40 Revicki DA, Willian MK, Menter A, Saurat JH, Harnam N, Kaul M. Relationship between clinical response to therapy and health-related quality of life outcomes in patients with moderate to severe plaque psoriasis. Dermatology. 2008;216(3):260-70.

41 Takeshita J, Callis Duffin K, Shin DB, Krueger GG, Robertson AD, Troxel AB, et al. Patientreported outcomes for psoriasis patients with clear versus almost clear skin in the clinical setting. J Am Acad Dermatol. 2014;71(4): 633-41.

42 Viswanathan HN, Chau D, Milmont CE, Yang W, Erondu N, Revicki DA, et al. Total skin clearance results in improvements in health-related quality of life and reduced symptom severity among patients with moderate to severe psoriasis. J Dermatolog Treat. 2015;26(3):235-9.

43 Uhlenhake EE, Kurkowski D, Feldman SR. Conversations on psoriasis - what patients want and what physicians can provide: a qualitative look at patient and physician expectations. J Dermatolog Treat. 2010;21(1): 6-12.

44 Warren RB, Gooderham M, Burge R, Zhu B, Amato D, Liu KH, et al. Comparison of cumulative clinical benefits of biologics for the treatment of psoriasis over 16 weeks: Results from a network meta-analysis. J Am Acad Dermatol. 2020;82(5):1138-49.

45 Krakowski P, Gerkowicz A, Pietrzak A, Krasowska D, Jurkiewicz A, Gorzelak M, et al. Psoriatic arthritis - new perspectives. Arch Med Sci. 2019;15(3):580-9.

46 Raychaudhuri SP, Wilken R, Sukhov AC, Raychaudhuri SK, Maverakis E. Management of psoriatic arthritis: early diagnosis, monitoring of disease severity and cutting edge therapies. J Autoimmun. 2017;76:21-37.

47 Haroon M, Gallagher P, FitzGerald O. Diagnostic delay of more than 6 months contributes to poor radiographic and functional outcome in psoriatic arthritis. Ann Rheum Dis. 2015;74(6):1045-50.

48 Girolomoni G, Gisondi P. Psoriasis and systemic inflammation: underdiagnosed enthesopathy. J Eur Acad Dermatol Venereol. 2009; 23(Suppl 1):3-8. 